\title{
Diabetic nephropathy in mice is aggravated by the absence of podocyte IRE1 and is correlated with reduced kidney ADH1 expression
}

\author{
Liping Xie ${ }^{1 \#}$, Kaifeng Guo ${ }^{1,2,3}$, Sijia Lu ${ }^{1 \#}$, Ning Wang", Yanping Wang ${ }^{1}$, Haibing Chen ${ }^{1}$ Junli Liu ${ }^{1}$, \\ Weiping $\mathrm{Jia}^{1}$
}

${ }^{1}$ Shanghai Diabetes Institute, Department of Endocrinology and Metabolism, Shanghai Jiao Tong University Affiliated Sixth People's Hospital, Shanghai Jiao Tong University School of Medicine, Shanghai, China; ${ }^{2}$ Department of Endocrinology and Metabolism, Minhang Hospital, Fudan University; ${ }^{3}$ Minhang Branch, Zhongshan Hospital, Fudan University, Central Hospital of Minhang District, Shanghai, China

Contributions: (I) Conception and design: W Jia, J Liu; (II) Administrative support: W Jia, J Liu; (III) Provision of study materials or patients: W Jia; (IV) Collection and assembly of data: L Xie, K Guo, S Lu; (V) Data analysis and interpretation: L Xie, K Guo, S Lu; (VI) Manuscript writing: All authors; (VII) Final approval of manuscript: All authors.

"These authors contributed equally to this work.

Correspondence to: Weiping Jia; Junli Liu. Shanghai Diabetes Institute, Department of Endocrinology and Metabolism, Shanghai Jiao Tong University Affiliated Sixth People’s Hospital, Shanghai Jiao Tong University School of Medicine, Shanghai, China. Email: wpjia@sjtu.edu.cn; liujunli@sjtu.edu.cn.

Background: Inositol-requiring enzyme 1 (IRE1) plays a critical role in attenuating endoplasmic reticulum (ER) stress associated with renal injury which may also be a factor in diabetic nephropathy (DN). Alcohol dehydrogenase type I (ADH1) activity is prominent in the kidney, ADH1 activity is also reported to exert protective effects against ER stress that are not caused by alcohol consumption. However, the role of IRE1 in $\mathrm{DN}$ and the correlation between IRE1 and ADH1 activity remain unclear.

Methods: IRE $1 \alpha$ floxed mice (Ire $1^{\text {fff }}$ ) of C57BL/6J background were established and crossbred with Ire $1 \alpha^{f f f}$ mice to produce podocyte-specific IRE1 $\alpha$ knockout mice. Male db/db mice (C57BLKS/J-leprdb/ leprdb mice) were used as a DN model. Male mice were made diabetic by injection of streptozotocin. pLKO.1-based vectors encoding short hairpin RNA (shRNA) specific to the IRE1 $\alpha$ gene were transfected into HEK293T cells to knockdown IRE1 $\alpha$ in mouse podocytes. ELISA, Masson's staining, and electron microscopy were performed to analyze the development of DN. The ADH1 expression was assayed by qPCR and western blot.

Results: We found that IRE activity was increased in the glomeruli of DN mouse models. In contrast, $\mathrm{ADH} 1$ expression was decreased in these models and mice with podocyte-specific disruption of IRE1 (PKO mice). PKO mice that were made diabetic using streptozotocin exhibited accelerated proteinuria, enhanced glomerular fibrosis, and podocyte cell death. In addition, in cultured podocytes, the knockdown of IRE1 downregulated the ADH1 mRNA expression and induced ER stress, consistent with the result of PKO mice, while its detrimental effects were reversed by ADH1 overexpression.

Conclusions: Activation of IRE1 in podocytes serves to limit the progress of DN. The dependence of kidney ADH1 expression on podocyte IRE1 further suggests that ADH1 activity may play an important role downstream of IRE1 in protecting against DN.

Keywords: Inositol-requiring enzyme 1 (IRE1); alcohol dehydrogenase type I (ADH1); podocyte; diabetic nephropathy (DN); albuminuria

Submitted Sep 10, 2020. Accepted for publication Feb 04, 2021.

doi: $10.21037 /$ atm-20-6356

View this article at: http://dx.doi.org/10.21037/atm-20-6356 


\section{Introduction}

The endoplasmic reticulum (ER) plays a critical role in protein folding, lipid synthesis, and calcium storage and release (1). Acute nutrient excess or obesity leads to the development of ER stress, triggering the activation of a group of signaling pathways, termed the unfolded protein response (UPR), in the liver, kidneys (2), and other organs. Three ER-stress-activated enzymesinositol-requiring enzyme 1 (IRE1 $\alpha$ ), PKR-like ER kinase (PERK), and activating transcription factor 6 (ATF6)together maintain ER homeostasis by activating distinct branches of the UPR. Of these enzymes, IRE1 $\alpha$ is the most evolutionarily conserved across eukaryote species (3). In the presence of adaptive ER stress, IRE1 $\alpha$ interaction with binding immunoglobulin protein (Bip) s relieved, and IRE $1 \alpha$ is further activated through oligomerization and autophosphorylation (4). Endoribonuclease activity possessed by activated IRE1 results in splicing of the messenger ribonucleic acid (mRNA) encoding X-box binding protein 1 (Xbp1), which activates transcriptiondependent processes that reduce ER stress, including IRE1dependent mRNA decay (RIDD) (5). Under cytotoxic ER stress, sustained IRE1 $\alpha$ ribonuclease (RNase) activity causes caspase-2-dependent apoptosis via the RIDD pathway (6). Furthermore, through IRE1A-mediated RIDD, thioredoxin-interacting protein (TXNIP) is induced to activate the NLR Family Pyrin Domain Containing 3 (NLRP3) inflammasome followed by interleukin (IL)$1 \mathrm{~b}$ secretion (7). Also, IRE1 $\alpha$ may serve as a complex with tumor necrosis factor (TNF) receptor-associated factor 2 (TRAF2) and apoptosis signal-regulating kinase 1 (ASK1) to initiate the c-Jun NH2-terminal kinase (JNK) pathway (8). Taken together, these processes could lead to programmed cell death. In contrast to normal physiology, in obesity and diabetes animal models, hepatic IRE1 $\alpha$ is S-nitrosylated, resulting in its impaired function and uncontrolled hepatic ER stress (9). ER stress may also be a factor in diabetic nephropathy (DN). Podocyte-specific disruption of IRE $1 \alpha$ in mice has resulted in injured podocytes and acute glomerulonephritis (10). However, whether deletion of the IRE $1 \alpha$ increases the susceptibility of podocytes to hyperglycemia-induced ER stress during diabetes remains unknown, along with the mechanism(s) through which IRE1 $\alpha$ disruption promotes podocyte injury. Thus, we investigated the interaction of podocyte-specific IRE $1 \alpha$ disruption with DN.

Alcohol dehydrogenase (ADH) is a key enzyme involved in converting ethanol into acetaldehyde, which is subsequently converted to acetic acid by aldehyde dehydrogenases. Five classes of ADH have been identified in humans, with a prominent role for class I ADH (ADH1) in ethanol metabolism. ADH1 consists of three subunits, with the $\alpha$ subunit being encoded by $A D H 1 A, A D H 1 B$, and $A D H 1 C$ genes (11). Interestingly, $\mathrm{ADH} 1$ has been shown to exert protective effects against diseases that are not caused by alcohol consumption (12). It was also reported that $\mathrm{ADH}$ reduces toxicity caused by lipid peroxidation products such as 4-hydroxynonenal (4 HNE) (13). Transgenic overexpression of $\mathrm{ADH} 1$ in the mouse heart blocks aginginduced down-regulation of $\mathrm{Ca}^{2+}$ cycling proteins and alleviates aging-associated diastolic dysfunction (14).

Moreover, $\mathrm{ADH}$ activity was reported to protect against ER stress-induced myocardial contractile dysfunction by relieving oxidative stress (12). Gene sets/pathways analysis has shown that $\mathrm{ADH} 1$ (ADH1B in particular) was significantly enriched in Gene Ontology (GO) categories, potentially related to obesity and insulin resistance. Also, the expression of ADH1B in human adipose tissues was downregulated in obese groups compared with the controls (15). However, an elevated ADH1 protein expression level was observed in the livers of non-alcoholic steatohepatitis patients (16). Overall, the evidence above supports the important role of $\mathrm{ADH} 1$ in the development of metabolic diseases. Although $\mathrm{ADH}$ activity is prominent in the kidney, whether and how renal ADH enzymes are altered during obesity and diabetes and how such changes may relate to the presence of ER stress have not yet been established. In this study, we found that normal ADH1 expression in the kidneys of mice required the presence of IRE1.

We present the following article in accordance with the ARRIVE reporting checklist (available at http://dx.doi. org/10.21037/atm-20-6356).

\section{Methods}

\section{Animal models}

Seven-week-old male C57BL/6J (C57) mice were purchased from the Model Animal Research Center of Nanjing University (Nanjing, China). The mice had unrestricted access to food and were kept under a $12 \mathrm{~h}$ light/dark cycle with controlled humidity $(55 \% \pm 5 \%)$ and temperature $\left(24 \pm 1^{\circ} \mathrm{C}\right)$. After 1 week of adaptation, male mice were made diabetic by intraperitoneal (ip) injection of streptozotocin (STZ, $50 \mathrm{mg} / \mathrm{kg}$, dissolved in $0.1 \mathrm{~mol} / \mathrm{L}$ citrate buffer, $\mathrm{pH}$ 4.5) for 5 days (17). Control mice were injected with the citrate buffer. Seven days after the last injection, only mice 
with glucose levels of $16.7 \mathrm{mmol} / \mathrm{L}$ were included in this study.

Male $d b / d b$ mice (C57BLKS/J-leprdb/leprdb mice) were used as a DN model, with age- and sex-matched $\mathrm{db} / \mathrm{dm}$ mice serving as controls (both from Nanjing University). All animal experiments were approved by the animal care committee of Shanghai Jiao Tong University Affiliated Sixth People's Hospital (project license No. 2017-0151), in compliance with the national institutes of Health guidelines for the care and use of animals.

\section{Creation of mice with deletion of IRE1 1 in podocytes}

IRE1 $\alpha$ floxed mice (Ire $1^{\text {ff }}$ ) of C57BL/6J background were kindly provided by Dr. Yong Liu (Wuhan University, Wuhan, China). Podocin-Cre transgenic mice (18), which express Cre recombinase under the podocin promoter's control, were backcrossed for six generations to C57BL/6J mice and then intercrossed with Ire $1 \alpha^{f f f}$ mice to produce podocyte-specific IRE1 $\alpha$ knockout mice (IRE1 ${ }^{\mathrm{PKO}}$ mice).

\section{Urine analysis}

Urine was collected from individual mice housed in metabolic cages for 12 hours and was centrifuged at 2,000 g for $5 \mathrm{~min}$. Urinary albumin concentrations were measured using a mouse albumin enzyme-linked immunosorbent assay (ELISA) kit (AKRAL-121; Shibayagi, Gunma, Japan) (19). Urinary creatinine concentration determination was conducted using the sarcosine oxidase PAP method on a Hitachi 7600 analyzer. The urine albumin-to-creatinine ratio (UACR) was detected during the experiments.

\section{Histopathological analysis and immunobistochemistry}

Kidney sections were stained with periodic acid-Schiff (PAS) for the estimation of glomerulosclerotic injury and mesangial expansion. Masson's trichrome staining was used for the assessment of glomerular extracellular matrix (ECM) accumulation. Glomerular cell apoptosis was evaluated with immunohistochemistry for cleaved caspase- 3 in each group of mice.

\section{Electron microscopy}

Small renal cortex pieces were fixed in $2.5 \%$ glutaraldehyde, followed by $1 \%$ osmium tetroxide, dehydrated, and then embedded in Epon 812. Ultrathin sections were examined using a transmission electron microscope. We performed a quantitative analysis of podocyte effacement according to the literature's methods (20). ImageJ software was used for image data analysis.

\section{Cell culture}

Mouse podocyte cells were maintained at $33{ }^{\circ} \mathrm{C}$ in Roswell Park Memorial Institute (RPMI)-1640 medium containing $100 \mathrm{U} / \mathrm{mL}$ interferon gamma (IFN $\gamma$ ) and $10 \%$ fetal bovine serum. The cells were transferred to a $37{ }^{\circ} \mathrm{C}$ incubator to induce differentiation for 10 days, as previously described (17). Culture media (RPMI-1640 media containing $5.5 \mathrm{mM}$ D-glucose) supplemented with D-glucose (final concentration $30 \mathrm{mM}$ ) was used as high glucose culture media. Culture media containing 5.6 $\mathrm{mM} \mathrm{D}$-glucose and $24.4 \mathrm{mM}$ D-mannitol was used as the control medium.

\section{Stable knockdown of IRE1 $\alpha$ in mouse podocytes}

For lentivirus packaging, pLKO.1-based vectors encoding short hairpin RNA (shRNA) specific to the IRE1 $\alpha$ gene were transfected into HEK293T cells. Plasmid psPAX2 and envelope plasmid pMD2.G were packaged into lentivirus using Lipofectamine 3000 Reagent (Invitrogen). For transduction of podocytes, cells were incubated with viral supernatants, added with $8 \mu \mathrm{g} / \mathrm{mL}$ polybrene, and then incubated for 24 hours using puromycin $(1 \mu \mathrm{g} / \mathrm{mL})$, with an additional 72 hours for selection.

\section{Statistical analysis}

The data are expressed as mean \pm standard error of the mean (SEM). The unpaired $t$-test was used to analyze data when two groups were present. For multiple group comparisons, analysis of variance (ANOVA) followed by Bonferroni correction was used. Statistical analyses were performed using GraphPad Prism software. Between-group differences in means were considered statistically significant when $\mathrm{P}<0.05$.

\section{Results}

\section{ADH1 expression is reduced in kidneys of diabetic mice and in high glucose-treated mouse podocytes.}

Previously, it was shown that ADH1 expression in human adipose is inversely correlated with body mass index (BMI) and fasting plasma insulin (15), which are two measures of 
obesity and insulin resistance. However, the relationship between obesity or diabetes and $\mathrm{ADH} 1$ expression in the kidney has remained unclear. To address this problem, we investigated renal ADH1 expression in two mouse models of DN standard models: STZ-induced diabetic mice, which model insulin-insufficient type I diabetes; and genetically obese $d b / d b$ mice, which model insulin-resistant type II diabetes. Using quantitative reverse transcription polymerase chain reaction (RT-PCR), we found that $\mathrm{ADH} 1$ mRNA levels were markedly reduced in the kidneys of STZ-induced DN mice (Figure 1A), as well as in the kidneys of $d b / d b$ DN mice (Figure 1B). Moreover, we found that in immortalized mouse renal podocyte cells (MPCs), exposure to high glucose concentration suppressed ADH1 mRNA in a time-dependent manner (Figure 1C), with long-term high glucose exposure producing MPC cell death (Figure 1D). These results indicate that hyperglycemia and DN development in mice are associated with down-regulation of renal ADH1 expression.

Next, we used immunoblotting to investigate the development of ER stress (UPR pathway activation) within the kidneys of diabetic $d b / d b$ mice. Abundances of phosphorylated IRE1 $\alpha$ (p-IRE1 $\alpha$ ) and PERK substrate EIF2 (p-EIF2) were increased in the kidneys of $d b / d b$ mice versus the non-diabetic controls (Figure $1 E, F$ ). In line with this, we found that concentrations of both Bip and cleaved ATF6 were also increased in the kidneys of $d b / d b$ mice (Figure 1E,F). Together, these results show an increase in renal ER stress of diabetic $d b / d b$ mice compared to their reduced renal $\mathrm{ADH} 1$ expression.

\section{Genetic disruption of IRE1a in podocytes accelerates the \\ development of DN in STZ-induced diabetic mice}

As described elsewhere, loss of IRE1 leads to unrelieved ER stress and severe ER dysfunction (21,22). ER function is crucial for podocytes to maintain protein homeostasis (23). To investigate ER stress's consequences during the development of DN, we crossed IRE $1^{f o x} f f b x$ mice with mice expressing Cre recombinase under the podocin promoter's control, producing podocyte-specific IRE1 knockout mice (or 'PKO mice'). The PKO and control (flox/flox) mice were challenged with STZ injection to induce diabetes and followed for 12 days or 3 months (12 weeks) to evaluate the effects of IRE1 deficiency on kidney function and histomorphology (Figure $2 A, B, C, D, E, F, G, H, I)$ (24).

Disruption of IRE1 did not, by itself, affect the body weights (Figure 2B,H) or kidney weights (Figure 2C,I) of the mice at either 12 days or 3 months follow-up. Furthermore, although STZ treatment profoundly elevated blood glucose in both $\mathrm{PKO}$ and control mice during the first 12 days post-injection (Figure 2D), STZ did not significantly affect the body or kidney weights during this period (Figure $2 B, C$ ). Importantly, although glucose levels became similarly elevated in $\mathrm{PKO}$ and control mice 12 days after STZ injection, the mean UACR (which is indicative of proteinuria) in PKO mice was greatly increased relative to that in the flox controls (Figure 2E). Thus, we concluded preliminarily that loss of podocyte IRE 1 and resultant podocyte ER stress was sufficient to accelerate $\mathrm{DN}$.

To confirm and extend these findings, similar investigations were repeated in the second cohort of $\mathrm{PKO}$ and control mice that we followed for 3 months after STZ injection (Figure 2F, G,H,I). As expected, mean blood glucose concentrations in the STZ-treated PKO and control mice increased dramatically within the first two weeks after STZ injection and closely paralleled one another after that (from 2 to 12 weeks) (Figure $2 F$ ). Similarly, the UACR became significantly ( two-fold) elevated in both PKO and control floxed mice by 2 weeks after STZ treatment. However, while the UACR in PKO mice continued to rise through the end of the study at 12 weeks, the UACR in the control flox/ flox mice remained at a lower 'plateau' level (Figure 2G). Thus, $\mathrm{DN}$-associated proteinuria was both aggravated and accelerated over the 3-month experiment by disruption of podocyte IRE1. Consistent with previous reports, body weights were lower in STZ-treated mice compared to the control (untreated) PKO and control mice, owing to polyuria and nutrient loss driven by diabetic hyperglycemia (Figure 2H) (25). Regardless, kidney weight to body weight ratios was higher in the STZ-treated PKO mice than in the controls (Figure 2I), suggesting greater renal damage in the PKO mice.

Three months after STZ injection, the mice's kidneys were harvested, and gross tissue morphology was assessed by hematoxylin and eosin (H\&E) staining (Figure 27). While IRE1 deletion did not, by itself, have an obvious effect on the structure of the glomeruli and tubules, STZ treatment resulted in renal glomeruli that appeared larger in PKO mice than in control mice. In conjunction with enlarged glomeruli, PAS staining (indicative of mesangial expansion) and Masson's trichrome staining (indicative of fibrosis) each showed relative enrichment in the glomeruli of STZ-treated PKO mice versus the STZ-treated controls (Figure 27). Compatible with these data, immunostaining revealed elevated glomerular fibronectin in STZ-treated 
A

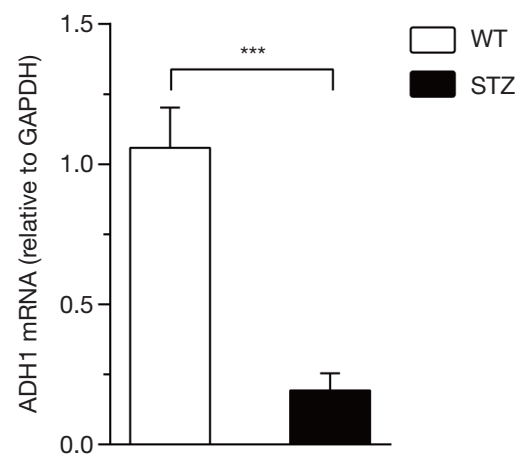

C

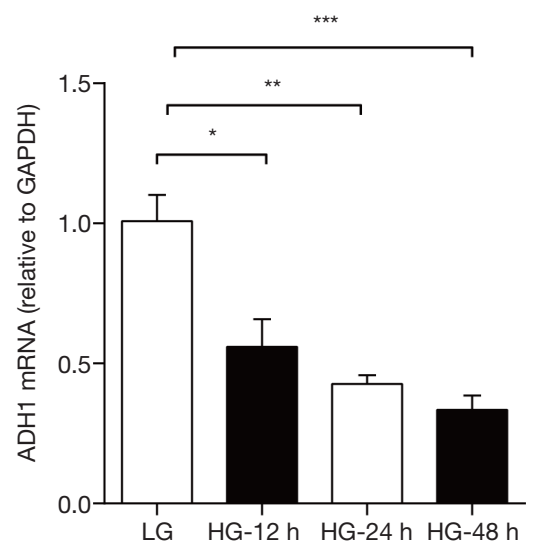

$\mathrm{E}$

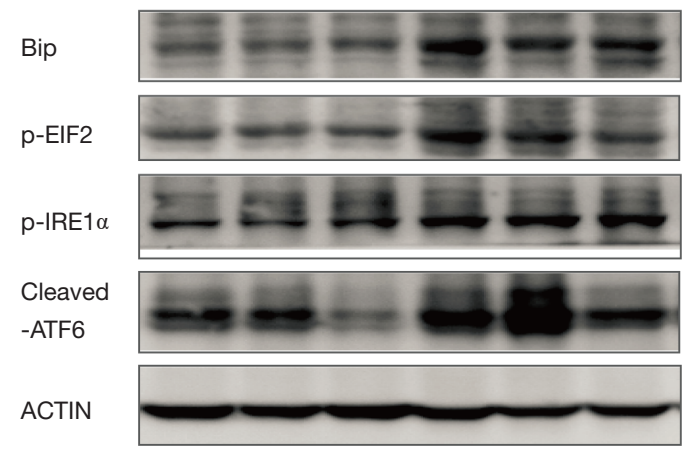

B

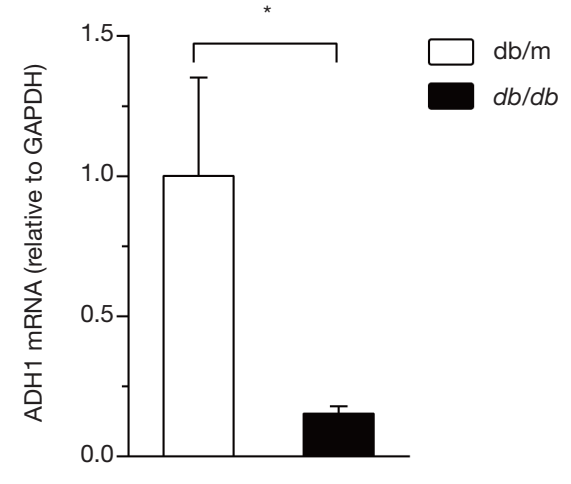

$\mathrm{D}$

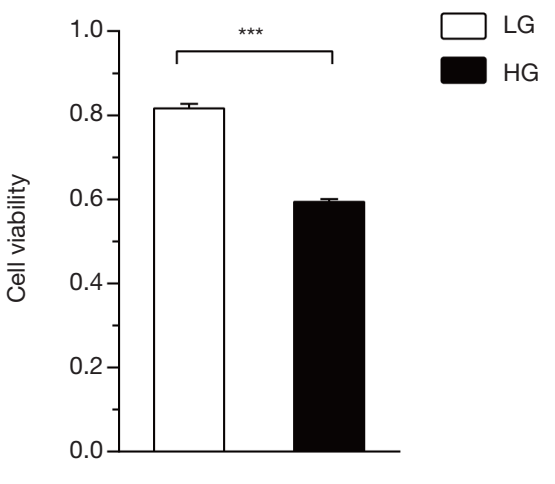

$\mathrm{F}$

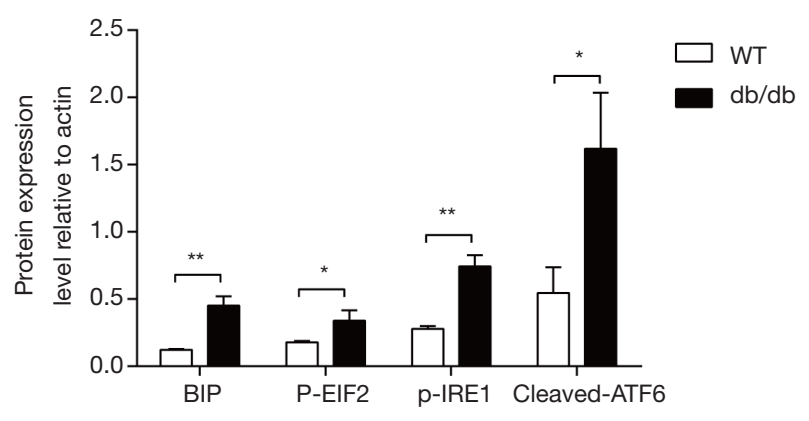

Figure $1 \mathrm{Adh} 1$ expression is reduced in both the renal glomeruli of diabetic mice and high glucose-treated mouse podocytes. (A) Quantitative RT-PCR assay for Adh1 mRNA in streptozotocin (STZ)-induced diabetic mice wild-type (WT) control mice. (B) Realtime RT-PCR assay for $A d b 1 \mathrm{mRNA}$ in $d b / d b$ mice and non-obese $d b / d m$ heterozygous control mice. (C) Quantitative RT-PCR analysis of Adh1 mRNA expression in immortalized mouse podocytes cultured under low glucose (LG) and high glucose (HG) conditions. (D) Cell viability of podocytes was detected by CCK-8 assay. (E) Western blot analysis of Bip, phosphorylated EIF2 (p-EIF2) and IRE1 (p-IRE1 $\alpha$ ), and cleaved ATF6 in $d b / d b$ and WT control mice. Equal protein loading was confirmed vy immunoblotting for $\beta$ actin. (F) Quantitation of western blot signals for Bip, p-EIF2, p-IRE1 $\alpha$, and cleaved-ATF6. Values are expressed as means $\pm \mathrm{SEM}$. ${ }^{*} \mathrm{P}<0.05,{ }^{* *} \mathrm{P}<0.01,{ }^{* *} \mathrm{P}<0.001$. 


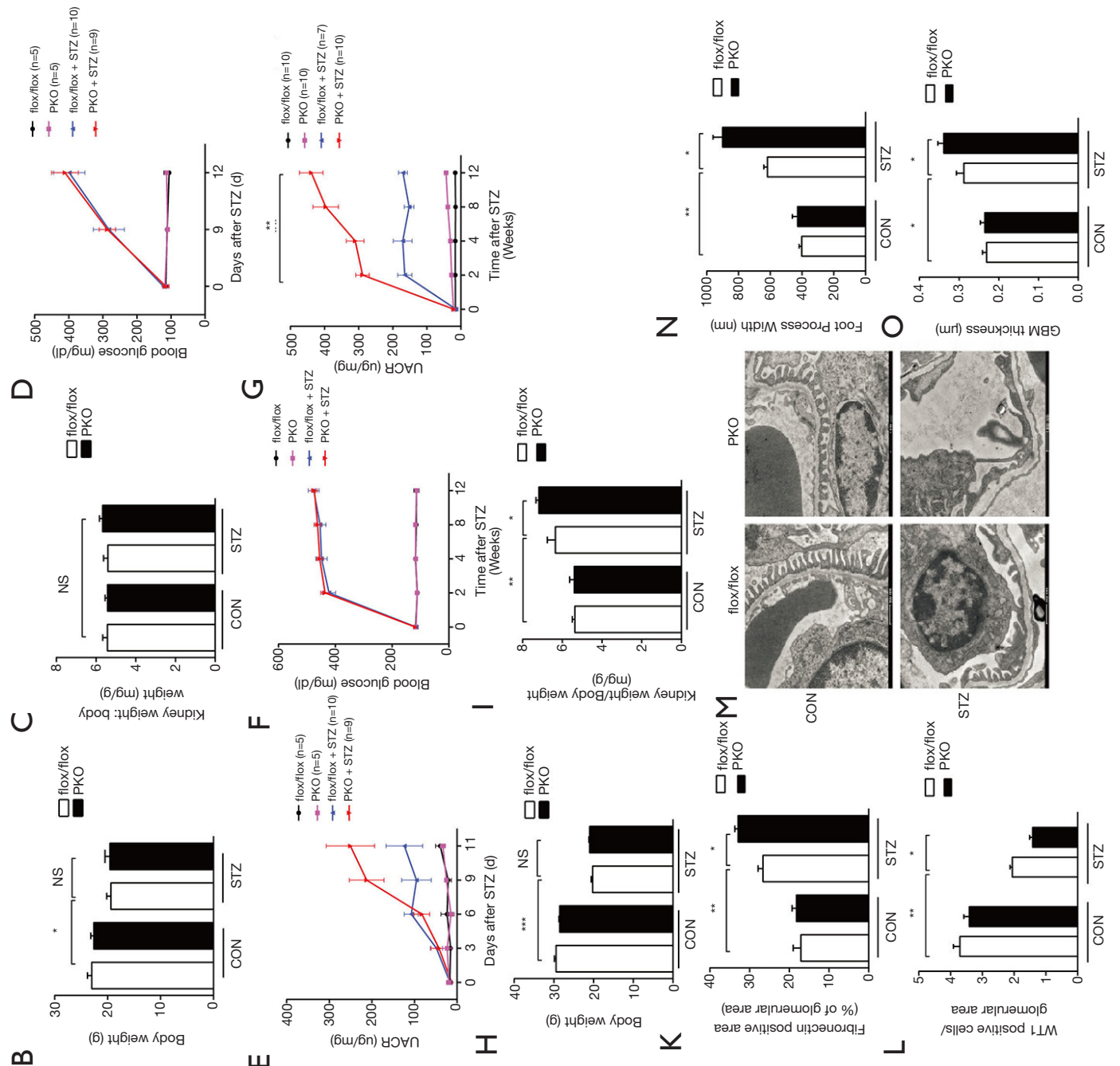

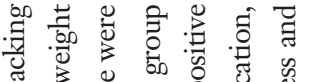

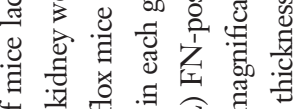
पे

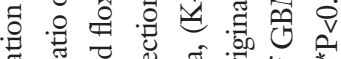
गั

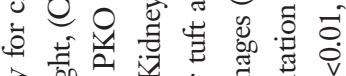
की

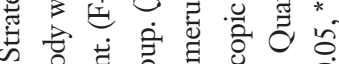

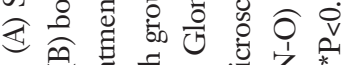

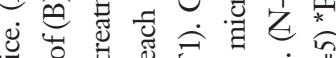

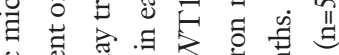

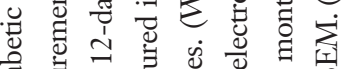
可 ठ

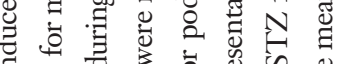

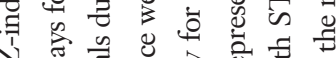

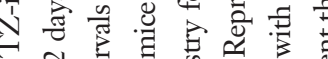
is 1 造

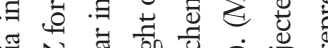
舀式焉

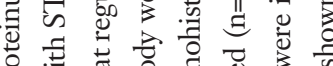

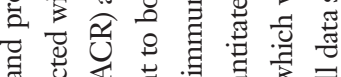

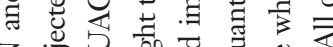

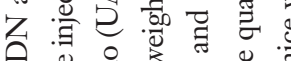
\&

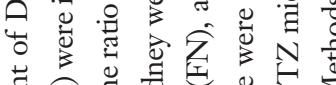

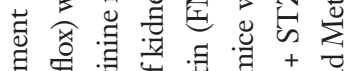

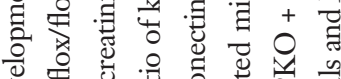

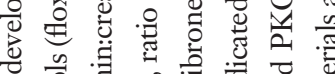

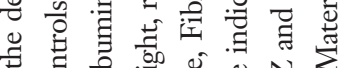

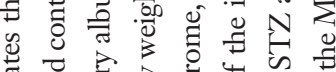

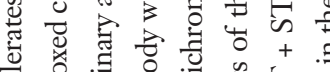

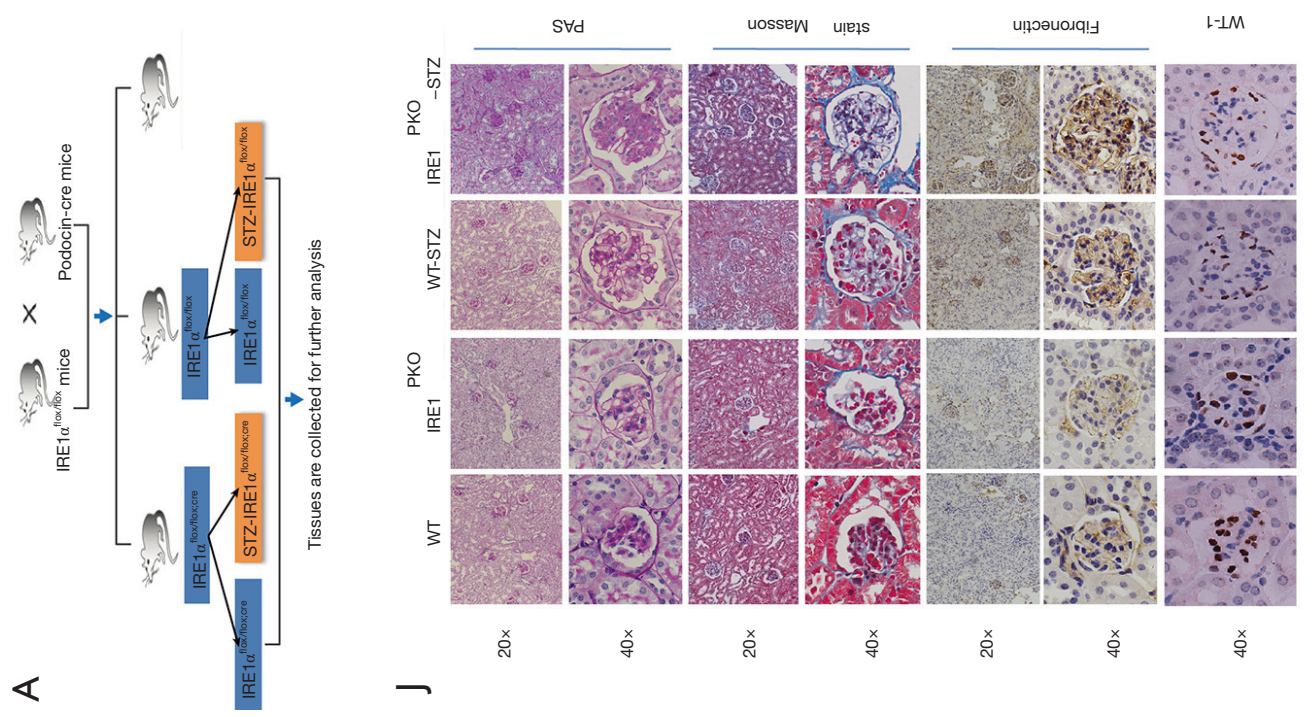

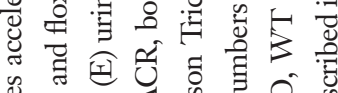

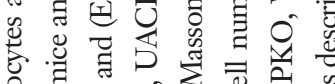
ठ

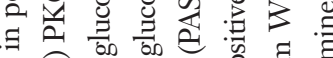

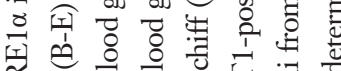

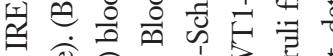
पे

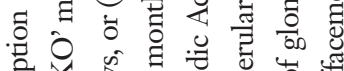

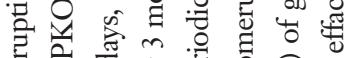

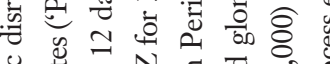

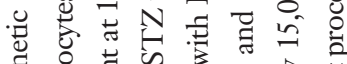

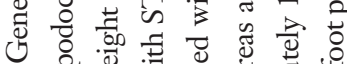
ن

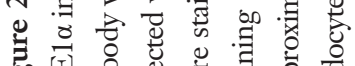

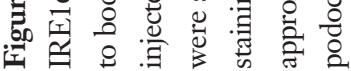


PKO mice (Figure 27). Quantitative analysis of fibronectin immunostaining showed that the fibronectin-positive area was significantly greater in the STZ-treated PKO mice than in the STZ-treated controls (Figure $2 K$ ). We also stained kidney sections with an antibody specific for podocyte marker WTI (Wilms Tumor 1); WT1 staining was clearly decreased in the STZ-treated PKO mice versus the STZ-treated control mice (Figure 27), as supported by quantitation of WT1-positive sectional areas (Figure 2L).

As revealed by electron microscopy, podocytes in STZtreated PKO mice exhibited severe foot process fusion and less ECM deposition than podocytes in STZ-treated control mice (Figure 2M). Consistently, quantitative analysis of Electron Microscopy (EM) sections revealed that podocytes in STZ-treated PKO mice had higher foot process width (Figure $2 \mathrm{~N}$ ) and glomerular basal membrane (GBM) thickness (Figure 2O) compared to podocytes in the STZ-treated flox/flox control mice. Collectively, these results demonstrate that knocking-down IRE1 deteriorates DN development.

\section{Podocyte-specific disruption of IRE1 $\alpha$ aggravates STZ- induced renal apoptosis}

During EM analysis (Figure $2 L$ ), we observed that podocytes in STZ-treated PKO mice became detached from the GBM and disappeared, suggesting that IRE1 disruption promoted podocyte cell death. Compatible with this hypothesis, immunoblotting showed that the concentration of cleaved caspase 3 was significantly higher in kidneys of STZ-treated PKO mice than in kidneys of STZ-treated control mice (Figure 3A, and signals quantitated in Figure 3B). Moreover, renal sections' immunostaining revealed stronger staining for cleaved caspase 3 in the STZ-treated PKO mice versus the STZ-treated controls (Figure 3C), which was supported by separate quantitative analysis of these data (Figure 3D). Together, these results document the occurrence of more severe glomerular cellular apoptosis in the STZ-treated PKO mice.

\section{Genetic deficiency or knockdown of podocyte IRE1 impairs ADH1 expression}

Since we observed lower ADH1 expression in the kidneys of DN mice (Figure 1A,B) - and IRE1 deficiency clearly accelerated $\mathrm{DN}$ in the $\mathrm{STZ}$ model—we hypothesized that IRE1 deficiency alone might reduce ADH1 expression.
Confirming this suspicion, we found by quantitative RTPCR and western blot that ADH1 mRNA and protein expression was both significantly lower in $\mathrm{PKO}$ mice than in flox/flox control mice, even in the absence of STZ treatment (Figure 4A,B). To gauge whether this result was attributable specifically to the absence of IRE1 in podocytes rather than to unknown systemic factors in PKO mice, we investigated whether reducing IRE1 expression in immortalized podocytes could also reduce ADH1 expression. Using a lentiviral shRNA vector, we knocked down the expression of IRE1 in cultured podocytes (Figure 4C) and found that ADH1 mRNA was correspondingly downregulated (Figure 4D). As expected from the knockdown of IRE1, the splicing of $\mathrm{Xbp} 1$ to $\mathrm{Xbp} 1 \mathrm{~s}$ mRNA in podocytes was also suppressed (Figure 4E). Cell division protein kinase 4 (Cdk4) and Cyclin D1 (Ccnd1), two major integrators of mitosis, were significantly suppressed (Figure 4F,G). We overexpressed ADH1 in cultured immortalized podocytes which were infected with lentivirus expressing shRNAs against IRE1 $\alpha(\mathrm{m} 428)$ or expressing a scrambled shRNA control. Western blot demonstrated that ADH1 overexpression showed reversing effects upon the increased of EIF2 (p-EIF2) (Figure 4H), which implied that ADH1 maybe was a direct downstream of IRE1 and it protects the kidney from hyperglycemia. These data demonstrate that, even in the absence of diabetes, the normal expression of $\mathrm{ADH} 1$ in podocytes depends on IRE1 function, which may have contributed to the enhanced severity of $\mathrm{DN}$ observed in STZ-treated PKO mice.

\section{Discussion}

ER stress is a pathologic component of various illnesses and reportedly contributes to DN by promoting podocyte apoptosis (26). The normal function of IRE1-a key ER transmembrane protein that initiates and regulates UPR signaling - is necessary for the maintenance of ER homeostasis (27). Herein, we investigated the effect of diminished IRE1 $\alpha$ function on STZ-induced DN in PKO mice, which lack IRE1 in podocytes. Detachment from the GBM and loss of podocytes was prominent in PKO mice's kidneys, and staining for activated (cleaved) caspase 3 was supportive of an ongoing apoptotic process. Concomitantly, the podocyte-specific disruption of IRE1 $\alpha$ in PKO mice accelerated the development of proteinuria and renal fibrosis. Thus, we conclude that activation of IRE1 and other UPR pathways protects against diabetes-related renal injury. 
A
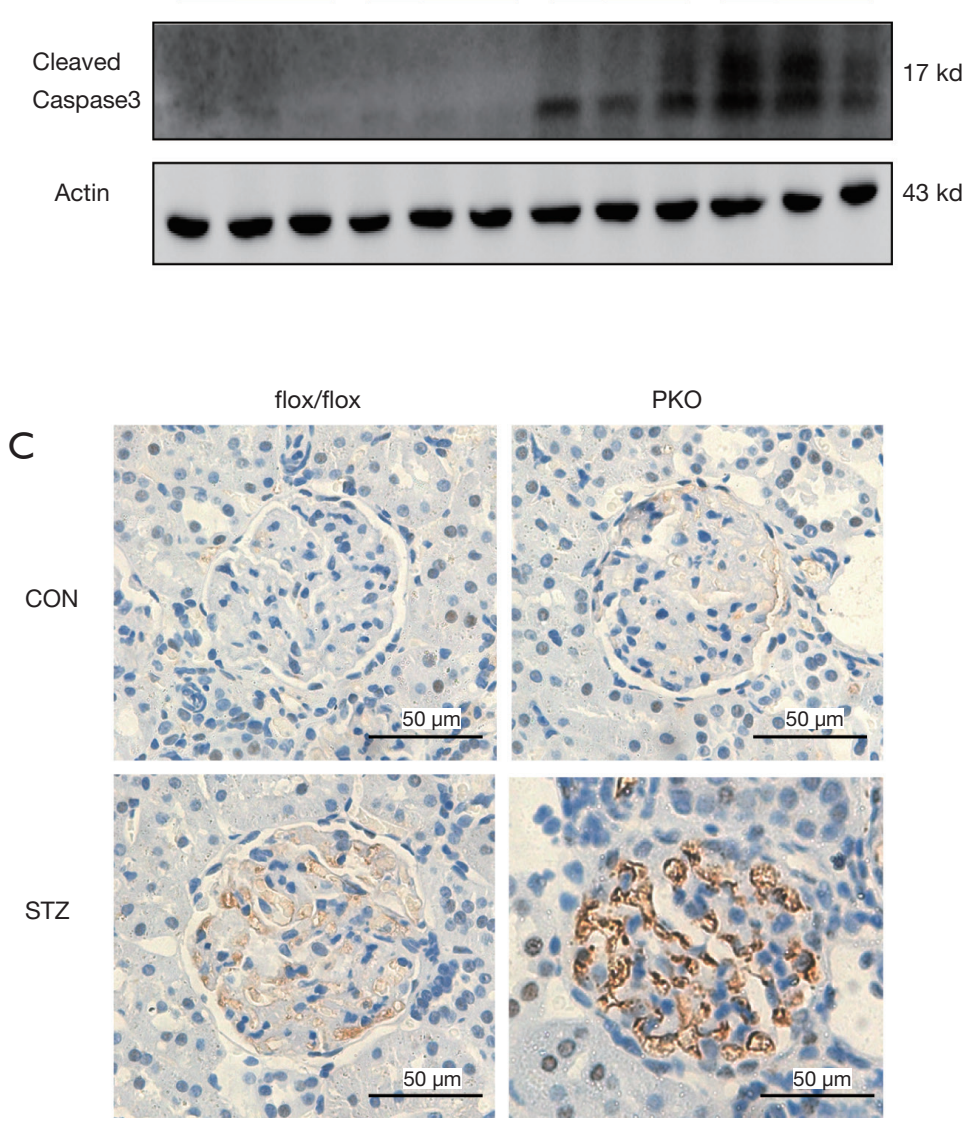

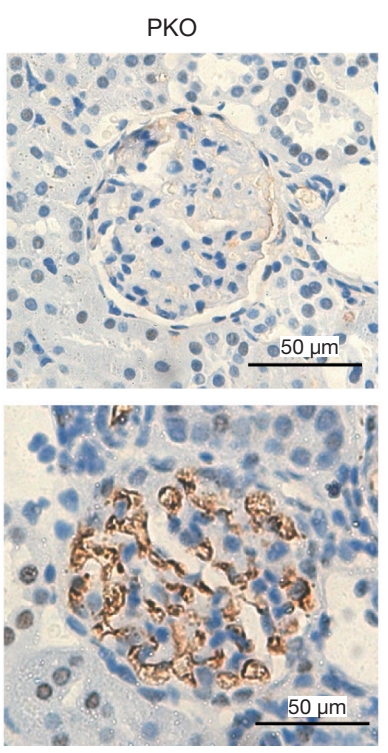

B
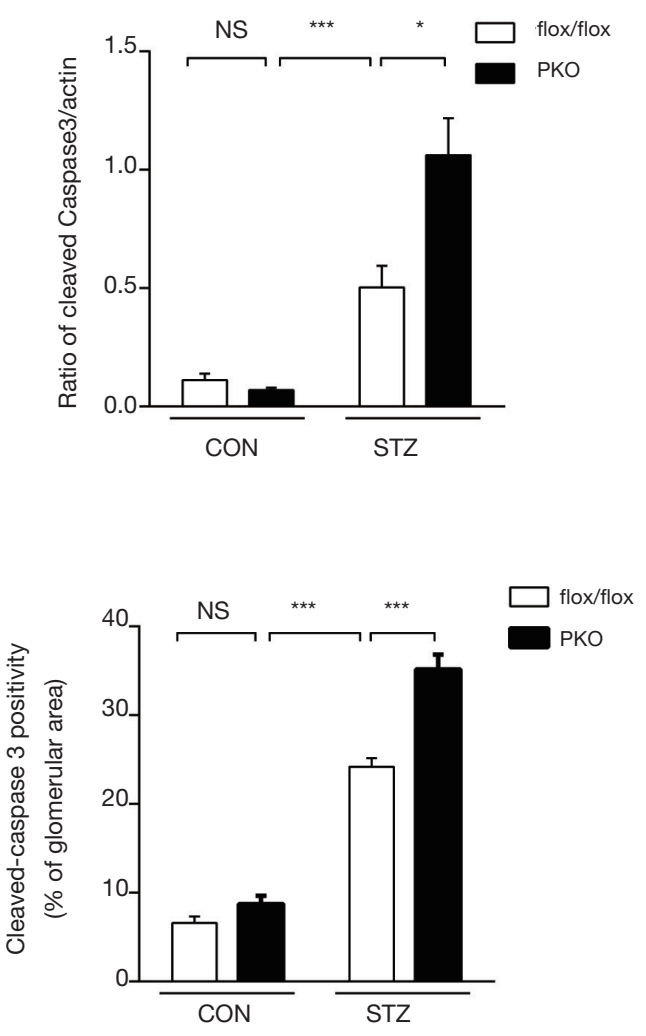

Figure 3 Podocyte-specific deletion of IRE1 $\alpha$ leads to aggravation of renal apoptosis in STZ-induced diabetic mice. (A) Western blotting analysis of cleaved (active) caspase 3 in isolated glomerulus lysates of mice in four groups. Equal protein loading was confirmed by staining with the actin antibody. (B) Quantitation of western blot bands for cleaved caspase 3. (C) Immunohistochemistry for activated caspase 3 were performed to determine the apoptosis of glomerular cells in each group of mice. (D) The percentage of positive staining was used to quantify cleaved caspase 3 , and the data are shown as mean \pm SEM $(n=5) .{ }^{*} \mathrm{P}<0.05,{ }^{* * *} \mathrm{P}<0.001$.

Attenuation of ER stress by activated IRE1 takes place through diverse and incompletely known pathways. For example, in addition to regulating Xbp1 splicing, IRE also regulates the expression of some cytokines and chemokines (28). In this study, we found that normal ADH1 expression in the mouse kidney also depends upon IRE1, and in cultured podocytes is strongly suppressed by high glucose conditions that model the diabetic state. Moreover, lower initial ADH1 expression in PKO mice was associated with accelerated proteinuria, worsened glomerular fibrosis, and enhanced apoptotic signaling during STZ-induced DN progress. Thus, it likely that preserved ADH1 activity at least partially underlies the protective function we observe for IRE1 against DN.
Although the apparent activation of IRE1 is higher in kidneys of STZ-induced and genetic $(d b / d b)$ DN model mice, ADH1 expression is nevertheless reduced. Pathways described in the context of other tissues could possibly explain these results. Under obese and diabetic conditions, obesity-related chronic inflammation has been shown to increase hepatic inducible nitric oxide synthase (iNOS), resulting in increased S-nitrosylation and diminished IRE1 function (9). Lower ADH1 expression in the kidneys of DN mice and high glucose-treated podocytes is compatible with the impairment of IRE1 function by this or another mechanism; however, confirmation of this hypothesis requires further investigation.

The underlying mechanisms of obesity-related 
A

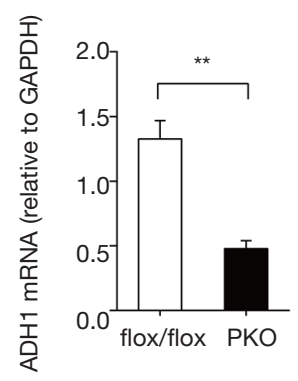

E

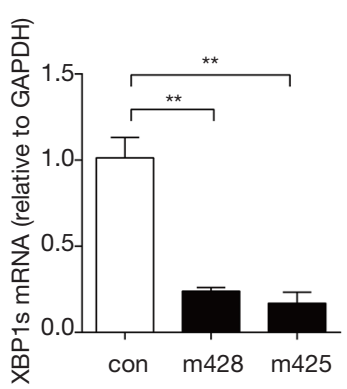

B

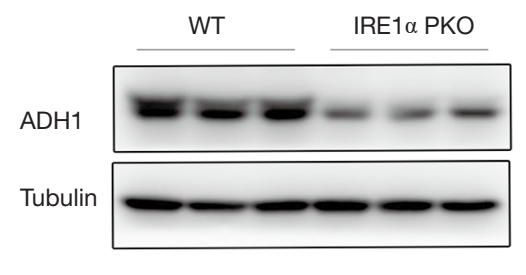

$\mathrm{F}$

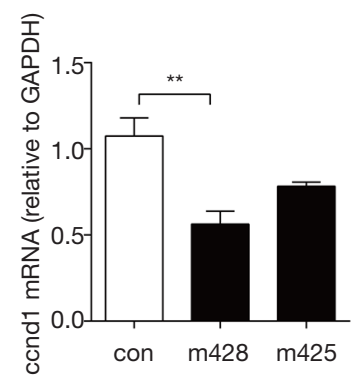

C

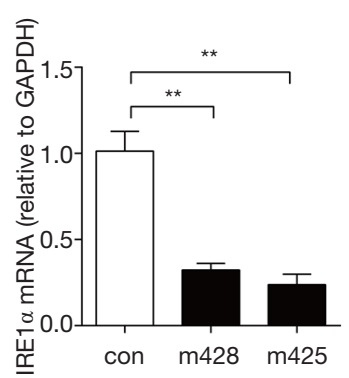

D

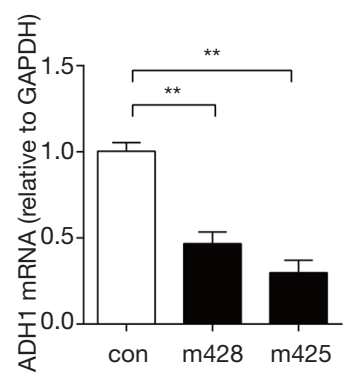

$\mathrm{H}$

Figure 4 Genetic deficiency or knockdown of podocyte IRE1 impairs Adb1 expression. (A) Quantitative RT-PCR assay for Adb1 mRNA quantity in WT and PKO mouse kidneys, normalized to the quantity of Gapdb mRNA. (B) Immunoblot of ADH1 in WT and PKO mouse kidneys. (C-G) Quantitative RT-PCR assay for Ern1 (IRE1 $\alpha$ ) mRNA, Adb1 mRNA, spliced Xbp1 (Xbp1s) mRNA, ccnd1 mRNA and cdk4 mRNA in immortalized podocytes following infection with lentivirus expressing shRNAs against IRE1 $\alpha$ (m428 and m425) or expressing a scrambled shRNA control (con). ( $\mathrm{n}=3$ for each group). ${ }^{*} \mathrm{P}<0.05,{ }^{* *} \mathrm{P}<0.01$. (H) Overexpressed ADH1 and the vector in $\mathrm{m} 428$ cells and con cells. Immunoblot of ADH1, IRE1 $\alpha$, p-EIF2 $\alpha$, EIF2a in $\mathrm{m} 428$ cells and con cells overexpressed with ADH1 or vector.

glomerulopathy are complex, while insulin resistance plays a pivotal role in this disease's pathogenesis. Previous studies have shown that insulin signaling modulates podocyte function and morphology (29). The increased sympathetic activation in obesity stimulates the renin-angiotensin system, resulting in insulin resistance (30). Insulin resistance resulting from insulin signaling interfering subsequently induces podocyte apoptosis, hypertrophy of the remaining podocytes, and glomerulosclerosis (31). In our study, reduced ADH1 was observed in genetically obese $\mathrm{db} / \mathrm{db}$ mice. The role of ADH1 in obesity-related glomerulopathy and the association between ADH1 and insulin resistance requires further investigation.

To our knowledge, a protective function of $\mathrm{ADH} 1$ in the kidney has not been reported. However, ADH1 has been reported to protect against ER stress-induced myocardial dysfunction (12). Our data shows that ADH1 expression is significantly impaired during the development of DN and is also generally dependent upon IRE1 function. These results provide novel insights into IRE1 and ADH1 as a core node in the ER stress response during the development of DN. Further elucidation of the mechanisms by which IRE1 regulates $\mathrm{ADH} 1$ expression and how $\mathrm{ADH} 1$ protects the kidney from hyperglycemia may open avenues for new therapeutic strategies against $\mathrm{DN}$.

$\mathrm{DN}$ is a vital diabetic complication, characterized by progressive GBM thickening and mesangial expansion. DN is, by far, the leading cause of end-stage renal disease (32) and an important predictor of mortality among Diabetes Melius patients (33). However, DN's clinically available therapies remain focused on inhibition of the reninangiotensin system, which does not reverse the disease but only decelerates its progression (34). To develop new potent drugs for DN, a better understanding of the molecular changes that contribute to $\mathrm{DN}$ development is required. Our findings have investigated the role of IRE1 and ADH1 
in $\mathrm{DN}$ and provided potential molecular targets that might be implemented in new drug development or could be used to guide the indication of specific therapeutic approaches.

Nonetheless, this research had several limitations that should be noted. Although we have identified the association between IRE1 and ADH1 in mouse podocytes of the DN model and explored the role of IRE1 and ADH1 in DN's pathophysiology, the exact interaction between IRE1 and ADH1 and the underlying mechanism by which $\mathrm{ADH} 1$ exerts its protective effects require further investigation.

\section{Acknowledgments}

We appreciate Dr. Yong Liu from Wuhan University for providing the IRE1 flox/flox mice. We appreciate AME editing service for language editing.

Funding: This work was supported by the National 973 project of China (2011CB504001), Natural Science Foundation of China (NSFC) major international (regional) joint research project (81220108006) and NSFC-NHMRC joint research grant (81561128016) to WJ. This study was also financially supported by the Training Program of the Major Research Plan of the National Natural Science Foundation of China (91857111), National Key R\&D Program of China (2018YFA0800600), National Science Fund for Excellent Young Scholars (31722028), National Natural Science Foundation of China (81770797), National Science Fund for Excellent Young Scholars (31722028) to JL. And was also financially supported by the Minhang District Health Committee (2020MZYS05), Shanghai Municipal Health Commission (20194Y0250) and National Natural Science Foundation of China (82000780) to KG.

\section{Footnote}

Reporting Checklist: The authors have completed the ARRIVE reporting checklist. Available at http://dx.doi. org/10.21037/atm-20-6356

Data Sharing Statement: Available at http://dx.doi. org/10.21037/atm-20-6356

Conflicts of Interest: All authors have completed the ICMJE uniform disclosure form (available at http://dx.doi. org/10.21037/atm-20-6356). The authors have no conflicts of interest to declare.
Ethical Statement: The authors are accountable for all aspects of the work in ensuring that questions related to the accuracy or integrity of any part of the work are appropriately investigated and resolved. All animal experiments were approved by the animal care committee of Shanghai Jiao Tong University Affiliated Sixth People's Hospital (project license No. 2017-0151), in compliance with the national institutes of Health guidelines for the care and use of animals.

Open Access Statement: This is an Open Access article distributed in accordance with the Creative Commons Attribution-NonCommercial-NoDerivs 4.0 International License (CC BY-NC-ND 4.0), which permits the noncommercial replication and distribution of the article with the strict proviso that no changes or edits are made and the original work is properly cited (including links to both the formal publication through the relevant DOI and the license). See: https://creativecommons.org/licenses/by-nc-nd/4.0/.

\section{References}

1. Park SW, Zhou Y, Lee J, et al. Sarco(endo)plasmic reticulum $\mathrm{Ca} 2+-\mathrm{ATP}$ ase $2 \mathrm{~b}$ is a major regulator of endoplasmic reticulum stress and glucose homeostasis in obesity. Proc Natl Acad Sci U S A 2010;107:19320-5.

2. Xie H, Huang L, Li Y, et al. Endoplasmic reticulum stress and renal lesion in mice with combination of high-fat diet and streptozotocin-induced diabetes. Acta Cir Bras 2016;31:150-5.

3. Yang J, Liu H, Li L, et al. Structural Insights into IRE1 Functions in the Unfolded Protein Response. Curr Med Chem 2016;23:4706-16.

4. Huang S, Xing Y, Liu Y. Emerging roles for the ER stress sensor IRE1 $\alpha$ in metabolic regulation and disease. J Biol Chem 2019;294:18726-41.

5. Hetz C, Martinon F, Rodriguez D, et al. The unfolded protein response: integrating stress signals through the stress sensor IRE1alpha. Physiol Rev 2011;91:1219-43.

6. Upton JP, Wang L, Han D, et al. IRE1 $\alpha$ Cleaves Select microRNAs During ER Stress to Derepress Translation of Proapoptotic Caspase-2. Science 2012;338:818-22.

7. Lerner AG, Upton JP, Praveen PV, et al. IRE1 $\alpha$ induces thioredoxin-interacting protein to activate the NLRP3 inflammasome and promote programmed cell death under irremediable ER stress. Cell Metab 2012;16:250-64.

8. Nishitoh H, Matsuzawa A, Tobiume K, et al. ASK1 is essential for endoplasmic reticulum stress-induced neuronal cell death triggered by expanded polyglutamine 
repeats. Genes Dev 2002;16:1345-55.

9. Yang L, Calay ES, Fan J, et al. METABOLISM. S-Nitrosylation links obesity-associated inflammation to endoplasmic reticulum dysfunction. Science 2015;349:500-6.

10. Kaufman DR, Papillon J, Larose L, et al. Deletion of inositol-requiring enzyme-1alpha in podocytes disrupts glomerular capillary integrity and autophagy. Mol Biol Cell 2017;28:1636-51.

11. Edenberg HJ. The genetics of alcohol metabolism: role of alcohol dehydrogenase and aldehyde dehydrogenase variants. Alcohol Res Health 2007;30:5-13.

12. Pang J, Fuller ND, Hu N, et al. Alcohol Dehydrogenase Protects against Endoplasmic Reticulum Stress-Induced Myocardial Contractile Dysfunction via Attenuation of Oxidative Stress and Autophagy: Role of PTEN-AktmTOR Signaling. PLoS One 2016;11:e0147322.

13. Sellin S, Holmquist B, Mannervik B, et al. Oxidation and reduction of 4-hydroxyalkenals catalyzed by isozymes of human alcohol dehydrogenase. Biochemistry 1991;30:2514-8.

14. Guo KK, Ren J. Cardiac overexpression of alcohol dehydrogenase $(\mathrm{ADH})$ alleviates aging-associated cardiomyocyte contractile dysfunction: role of intracellular Ca2+ cycling proteins. Aging Cell 2006;5:259-65.

15. Winnier DA, Fourcaudot M, Norton L, et al. Transcriptomic identification of ADH1B as a novel candidate gene for obesity and insulin resistance in human adipose tissue in Mexican Americans from the Veterans Administration Genetic Epidemiology Study (VAGES). PLoS One 2015;10:e0119941.

16. Baker SS, Baker RD, Liu W, et al. Role of alcohol metabolism in non-alcoholic steatohepatitis. PLoS One 2010;5:e9570.

17. Chen J, Chen JK, Harris RC. EGF receptor deletion in podocytes attenuates diabetic nephropathy. J Am Soc Nephrol 2015;26:1115-25.

18. Moeller MJ, Sanden SK, Soofi A, et al. Podocyte-specific expression of cre recombinase in transgenic mice. Genesis 2003;35:39-42.

19. Maki T, Maeda Y, Sonoda N, et al. Renoprotective effect of a novel selective PPARalpha modulator K-877 in db/db mice: A role of diacylglycerol-protein kinase C-NAD $(\mathrm{P}) \mathrm{H}$ oxidase pathway. Metabolism 2017;71:33-45.

20. Mallipattu SK, Gallagher EJ, LeRoith D, et al. Diabetic nephropathy in a nonobese mouse model of type 2 diabetes mellitus. Am J Physiol Renal Physiol 2014;306:F1008-17.

21. Shao M, Shan B, Liu Y, et al. Hepatic IRE1alpha regulates fasting-induced metabolic adaptive programs through the XBP1s-PPARalpha axis signalling. Nat Commun
2014;5:3528.

22. Kanekura K, Ma X, Murphy JT, et al. IRE1 prevents endoplasmic reticulum membrane permeabilization and cell death under pathological conditions. Sci Signal 2015;8:ra62.

23. Cybulsky AV. Endoplasmic reticulum stress, the unfolded protein response and autophagy in kidney diseases. Nat Rev Nephrol 2017;13:681-96.

24. Lee S, Rose'meyer R, McDermott C, et al. Diabetesinduced alterations in urothelium function: Enhanced ATP release and nerve-evoked contractions in the streptozotocin rat bladder. Clin Exp Pharmacol Physiol 2018;45:1161-9.

25. Furman BL. Streptozotocin-Induced Diabetic Models in Mice and Rats. Curr Protoc Pharmacol 2015;70:5.47.15.47.20.

26. Rong G, Tang X, Guo T, et al. Advanced oxidation protein products induce apoptosis in podocytes through induction of endoplasmic reticulum stress. J Physiol Biochem 2015;71:455-70.

27. Parmar VM, Schroder M. Sensing endoplasmic reticulum stress. Adv Exp Med Biol 2012;738:153-68.

28. Tufanli O, Telkoparan Akillilar P, Acosta-Alvear D, et al. Targeting IRE1 with small molecules counteracts progression of atherosclerosis. Proc Natl Acad Sci U S A 2017;114:E1395-404.

29. Welsh GI, Hale LJ, Eremina V, et al. Insulin signaling to the glomerular podocyte is critical for normal kidney function. Cell Metab 2010;12:329-40.

30. Bayliss G, Weinrauch LA, D'Elia JA. Pathophysiology of obesity-related renal dysfunction contributes to diabetic nephropathy. Curr Diab Rep 2012;12:440-6.

31. Yang S, Cao C, Deng T, et al. Obesity-Related Glomerulopathy: A Latent Change in Obesity Requiring More Attention. Kidney Blood Press Res 2020;45:510-22.

32. Webster AC, Nagler EV, Morton RL, et al. Chronic Kidney Disease. Lancet 2017;389:1238-52.

33. Barkoudah E, Skali H, Uno H, et al. Mortality rates in trials of subjects with type 2 diabetes. J Am Heart Assoc 2012;1:8-15.

34. Susztak K, Böttinger EP. Diabetic nephropathy: a frontier for personalized medicine. J Am Soc Nephrol 2006;17:361-7.

Cite this article as: Xie L, Guo K, Lu S, Wang N, Wang Y, Chen H, Liu J, Jia W. Diabetic nephropathy in mice is aggravated by the absence of podocyte IRE1 and is correlated with reduced kidney ADH1 expression. Ann Transl Med 2021;9(8):636. doi: 10.21037/atm-20-6356 